\title{
No Role for Chemoradiotherapy When Compared with Chemotherapy Alone in Elderly Patients with Localized Low Risk Aggressive Lymphoma: Final Results of the LNH93-4 GELA Study.
}

Georges Fillet ${ }^{1}$, C. Bonnet ${ }^{1, *}$, N. Mounier ${ }^{2, *}$, G. Ganem ${ }^{3, *}$, T. Molina ${ }^{4, *}$, C. Thieblemont ${ }^{5}$, C. Fermé $^{6}$, B. Quesnel ${ }^{7}$, C. Martin ${ }^{8, *}$, C. Gisselbrecht ${ }^{2}$, H. Tilly ${ }^{9}$ and F. Reyes ${ }^{10}$

${ }^{1}$ Hematology, CHU-Sart Tilman, Liège, Belgium; ${ }^{2}$ Hematology, Hôpital Saint-Louis, Paris, France; ${ }^{3}$ Radiotherapy, Clinique Victor Hugo, Le Mans, France; ${ }^{4}$ Pathology, Hôpital Hôtel Dieu, Paris, France; ${ }^{5}$ Hematology, Hôpital Lyon Sud, Lyon, France; ${ }^{6}$ Hematology, Institut Gustave Roussy, Villejuif, France; ${ }^{7}$ Hematology, CHU de Lille, Lille, France; ${ }^{8}$ Hematology, Centre Hospitalier d'Annecy, Annecy, France; ${ }^{9}$ Hematology, Centre Henri Becquerel, Rouen, France and ${ }^{10}$ Hematology, Centre Hospitalier Henri Mondor, Créteil, France.

\begin{abstract}
Chemoradiotherapy is standard treatment for localized aggressive lymphoma (Miller et al., NEJM, 1998). Because previously published series were heterogeneous with regard to prognostic factors such as age, we aimed to determine the optimal therapy for elderly patients with low risk localized lymphoma. From March 1993 to June 2002, 576 patients (pts) over 60y of age with aggressive lymphoma (WF histology: F, G, H or Kiel anaplastic) and without any adverse factor of the age-adjusted International Pronostic Index were randomly assigned to a chemoradiotherapy arm (299 pts) consisting of 4 cycles of CHOP (doxorubicin $50 \mathrm{mg} / \mathrm{m} 2 \mathrm{~d} 1$, cyclophosphamide $750 \mathrm{mg} / \mathrm{m} 2 \mathrm{~d} 1$, vincristine $1.4 \mathrm{mg} / \mathrm{m} 2$ [up to $2 \mathrm{mg}$ ] d1, prednisone $60 \mathrm{mg} / \mathrm{m} 2$ d1-5) given every 3 weeks followed by 40 Gy involved-field radiotherapy or to a chemotherapyalone arm (277 pts) consisting of 4 cycles of CHOP given every 3 weeks. Randomization was stratified according to center and the presence of bulky disease $(10 \mathrm{~cm})$. Principal characteristics were: median age, 68y; male gender, $52 \%$; stage I, 63\%; bulky disease, $8 \%$; extranodal involvement, 56\%; diffuse large B-cell histology, 80\%. Complete response at the end of treatment was similar in both groups ( $89 \%$ and $90 \%$ respectively). Treatment-related death occurred in $1 \%$ of pts in each group. On an intent-to-treat basis and with a median follow-up of $6.8 \mathrm{y}$, event-free survival (EFS) and overall survival (OS) did not differ significantly between the two treatment groups ( $p=0.7$ and $p=0.6$, respectively). $5 y$-EFS rates were $68 \%$ for pts treated with chemotherapy alone as compared to $66 \%$ for those receiving chemoradiotherapy; $5 y-O S$ rates were $72 \%$ and $68 \%$ respectively. $66 \%$, of deaths resulted from lymphoma progression. In the subgroup of $247 \mathrm{pts}$ over $70 \mathrm{y}$ of age, the $5 \mathrm{y}-\mathrm{OS}$ was higher in those treated by chemotherapy alone $(70 \%$ as compared to $58 \%)$ but this trend did not reach significance $(p=0.1)$. In a multivariate analysis of the 576 pts, EFS and OS were affected by stage II $(p<0.0001)$, male gender $(\mathrm{p}=0.02)$, not by tumor bulk.
\end{abstract}

Conclusion: mature data of the LNH93-4 trial indicate that CHOP plus radiotherapy does not provide any advantage over $\mathrm{CHOP}$ alone for the treatment of low risk localized aggressive lymphoma in elderly pts. To note, although the majority of patients had stage I disease, local control by irradiation did not decrease the overall relapse rate. A similar conclusion was recently reached in young adult pts treated by chemotherapy alone (Reyes et al, NEMJ 2005). Taking these results together, we consider that local irradiation following chemotherapy has no role in the treatment of pts with low risk localized aggressive lymphoma. On the basis of its previous 
published results (Coiffier et al, NEJM, 2002), the GELA recommends to treat such elderly pts with rituximab plus CHOP. 